\title{
Actitudes hacia la homoparentalidad: Validación psicométrica de dos escalas en una muestra de estudiantes mexicanos
}

\author{
Attitudes Towards Homoparentality: Psychometric Validation of two \\ Scales in a Sample of Mexican Students
}

\section{Atitudes em relação à homoparentalidade: validação psicométrica de duas escalas numa amostra de estudantes mexicanos}

\author{
Virginia Barragán-Pérez, MD., MSc. * \\ Shoshana Berenzon-Gorn, Ps., PhD. ** \\ Guadalupe Silvia García-De la Torre, MSc *** \\ María del Carmen Lara-Muñoz, MD., PhD. ****
}

\begin{abstract}
Resumen
Introducción: La legalización del llamado matrimonio igualitario, y de la adopción de menores por parejas homosexuales, es un fenómeno reciente y que debe ser estudiado, para lo cual es necesario contar con medidas confiables que permitan dar cuenta de cómo la sociedad percibe esta nueva configuración familiar. Objetivo: Determinar las propiedades psicométricas de dos escalas: Actitudes Frente a las Familias Homoparentales (AFFH); y Creencias Acerca del Ajuste de los Niños de Familias Homoparentales (CANFH). Metodología: La AFFH, con 20 reactivos, fue diseñada para dar cuenta de las actitudes hacia las familias homoparentales. La CANFH tiene 14 reactivos organizados en las subescalas de oposición individual (OI) y oposición normativa ( $\mathrm{ON})$. Ambas escalas tienen afirmaciones que deben ser respondidas con opciones tipo Likert que van del 1 totalmente de acuerdo, al 5 totalmente en desacuerdo. La CANFH fue aplicada a 170 estudiantes universitarios (78 (46\%) hombres, 92 (54\%) mujeres. Media
\end{abstract}

de edad: 18.4 DE+0.94), y la AFFH a 88 (35 (40\%) hombres, $53(60 \%)$ mujeres. Media de edad: $18.2 \mathrm{DE}+0.84)$. Se determinaron las propiedades psicométricas por medio de análisis factorial y de consistencia interna. Resultados: La AFFH resultó con un $\alpha=0.91$; los reactivos se organizaron en dos factores que explican $46.14 \%$ de la varianza. En el caso de la CANFH se confirmó la estructura factorial de dos subescalas explicativas de $65.49 \%$ de la varianza y con un $\alpha=0.94$. Conclusiones: Los datos indican que ambas escalas poseen las propiedades psicométricas adecuadas para medir las actitudes de estudiantes mexicanos hacia las familias homoparentales y hacia los efectos que estas puedan tener en los menores adoptados. [Barragán-Pérez $V$, Berenzon-Gorn S, García-De la Torre GS, Lara-Muñoz MDC. Actitudes hacia la homoparentalidad: Validación psicométrica de dos escalas en una muestra de estudiantes mexicanos. MedUNAB 2016; 19(2): 85-94].

Palabras clave: Homosexualidad; Responsabilidad parental; Actitud; Escalas; Psicometría; Estudiantes.

* Maestra en Ciencias, Departamento de Psiquiatría y Salud Mental, Facultad de Medicina, UNAM, México D.F., México.

** PhD en Psicología, Dirección de Investigaciones Epidemiológicas y Sociales, Instituto Nacional de Psiquiatría Ramón de la Fuente Muñiz, México D.F., México.

*** Maestra en Ciencias Sociomédicas (Epidemiología), Departamento de Salud Pública, Facultad de Medicina. UNAM, México D.F., México.

**** PhD en Ciencias Médicas, Facultad de Medicina, Benemérita Universidad Autónoma de Puebla, Heroica Puebla de Zaragoza, México.

Autor de correspondencia: Virginia Barragán Pérez, Circuito interior s/n Edificio F. Col. Ciudad Universitaria, UNAM, México, D.F. C.P. 04510. Tel: (55)56232130 Ext. 43136. Correo electrónico: virginiabarragan@unam.mx 


\section{Abstract}

Introduction: Same-sex marriage and adoption legalization constitute a growing and relatively new research field. Its study needs reliable and valid measures that allow to understand the society's perception about this new family order. Objective: Assess the psychometric properties of two scalesdevised to measure same-sex parenting attitudes: Attitudes toward Same-sex Families Scale (AFFH for its acronym in Spanish), and Scale on Beliefs about Children's Adjustment in Same-sex families (CANFH in Spanish). Methodology: AFFH scale is a 20 item measure designed to account for attitudes towards same-sex families. CANFH is a 14-item measure consisting of two scales, Individual Opposition (IO) and Normative Opposition (NO). Both are Likert scales with five response options (1. Strongly Agree to 5. Strongly Disagree). The CANFH was answered by 170 college students (78 (46\%) males, 92 (54\%) females; Average age: 18.4 SD 0.94 ), and the AFFH by 88 college students (35 (40\%) males, $53(60 \%)$ females, average age: 18.2 SD + 0.84). Results: AFFH items were organized into two factors that explained $46.14 \%$ of variance with a Cronbach's alpha of 0.91 . CANFH items were grouped in a two factors structure that explained $65.49 \%$ of the variance $(\alpha=0.94)$. Conclusions: CANFH and AFFH scales have adequate psychometric properties to measure Mexican college students' attitudes toward same-sex families and to assess the beliefs about children's adjustment in same-sex families. [Barragán-Pérez V, Berenzon-Gorn S, García-De la Torre GS, Lara-Muñoz MDC. Attitudes Towards Homoparentality: Psychometric Validation of two Scales in a Sample of Mexican Students. MedUNAB 2016; 19(2): 8594].

Keywords: Homosexuality; Parenting; Attitude; Scales; Psychometrics; Students.

\section{Introducción}

En los últimos años, el tema de las familias homoparentales ha cobrado vigencia y relevancia. El primer país que aceptó las uniones matrimoniales entre homosexuales fue Holanda en el año 2000, aunque el precedente lo estableció Dinamarca en 1989 al otorgar a las parejas homosexuales los mismos derechos maritales que los de las personas casadas, solo omitiendo el derecho de contraer nupcias en ceremonias religiosas (1). A partir de la legalización del matrimonio en Holanda, el asunto se empezó a discutir en más países (2), lo que nos ha llevado a que en la actualidad, de acuerdo con una nota publicada en el periódico El País en abril de 2016 (3), sean 24 países (Holanda, Bélgica, Dinamarca, España, Francia, Luxemburgo, Noruega, Irlanda, Suecia, Portugal, Grecia, Inglaterra, Gales, Escocia, Islandia, Estados Unidos, Canadá, Argentina, Uruguay, Brasil, México (varios estados), Sudáfrica, Nueva Zelanda y Colombia) en los que el matrimonio homosexual es legal. Si bien la aprobación del matrimonio homosexual supone un avance en materia de derechos humanos, aún quedan pendientes otros rubros fundamentales como el

\section{Resumo}

Introdução: A legalização do chamado casamento do mesmo sexo e adoção de crianças por casais homossexuais, é um fenômeno recente, que deve ser estudado, para a qual é necessário ter medidas confiáveis que permitem ver como a sociedade percebe esta nova configuração familiar. Objetivo: determinar as propriedades psicométricas de duas escalas: Atitudes para com as famílias homoparentais (AFFH); e as crenças sobre o equilíbrio das crianças de famílias homoparentais. Metodologia: O AFFH, com 20 itens, foi projetado para explicar atitudes das famílias homoparentais. $\mathrm{O}$ CANFH tem 14 reagentes organizados em sub-escalas de oposição individual (OI) e oposição regulamentar (ON). Ambas as escalas são declarações que devem ser respondidas com opções de tipo Likert, entre 1 que significa estar de acordo e 5 que discorda totalmente (CANFH). O CANFH foi aplicado a 170 estudantes universitários (78 (46\%) do sexo masculino, 92 (54\%) mulheres com idade média: $18.4+0.94)$, e AFFH a 88 (35 (40\%) do sexo masculino, $53(60 \%)$ mulheres média de idade. $18.2+0.84)$. Foram determinadas as propriedades psicométricas por meio de análise fatorial e consistência interna. Resultados: AAFFH foi $\alpha=0.91 \mathrm{com}$ reagentes foram organizados em dois fatores que explicam $46.14 \%$ da variância. No caso de a estrutura fatorial CANFH dois explicativa $65.49 \%$ da variância e um $\alpha=0,94$ subescalas foi confirmada. Conclusões: Os dados indicam que ambas as escalas têm o direito de medir as atitudes dos estudantes em relação homoparentais famílias mexicanas e para os efeitos que podem ter sobre as crianças adotadas propriedades psicométricas. [Barragán-Pérez V, Berenzon-Gorn S, Garcia-De la Torre GS, Lara-Muñoz MDC. Atitudes em relação à homoparentalidade: validação psicométrica de duas escalas em uma amostra de estudantes mexicanos. MedUNAB 2016; 19 (2): 85-94].

Palavras-chave: Homossexualidade; Poder familiar; atitude; Escalas; Psicometria; Estudantes.

derecho a tener hijos, mismo que se niega aduciendo que la crianza por parte de una pareja homosexual puede ser dañina para los menores (4). Al respecto, diversos autores, sobre todo estadounidenses y europeos, se han abocado a estudiar y comparar el desarrollo de los hijos de parejas heterosexuales y homosexuales y han reportado que no hay diferencias significativas entre ellos en cuanto a sus historias psiquiátricas $(5,6)$, niveles de ansiedad (7), conducta general $(8,9)$, desarrollo académico $(7,9)$, ajuste social $(10,11)$, orientación sexual $(5,10-15)$, e identidad de género $(10,11)$; y que la variable que resulta predictiva de la salud mental de los hijos, tanto de familias heteroparentales como de familias homoparentales es la calidad y cercanía entre los progenitores $(5-7,14-16)$.

En el mismo sentido, la Asociación Psicológica Americana concluye a partir de una revisión sistemática de más de cien artículos sobre paternidad en familias homoparentales de E.U.A y Europa, que tanto los hijos biológicos como los adoptados por parejas homosexuales: i. no presentan problemas psicológicos o sociales que puedan atribuirse a la orientación sexual de sus padres; ii. desarrollan patrones de 
género similares a los de hijos de padres heterosexuales; iii. su orientación sexual no depende de la de sus padres; y iv. tienen tantas posibilidades de tener problemas psicológicos o sociales, o de ser homosexuales, como las de sus iguales procedentes de familias heteroparentales (17).

Contexto mexicano: si bien la información sobre los efectos de la homoparentalidad es amplia y diversa, en Latinoamérica aún existe un rezago y desconocimiento sobre el tema. Aunque México es reconocido por ser uno de los pioneros en la legalización del matrimonio entre personas del mismo sexo, en 2009 se aprobó una ley que permite el matrimonio homosexual y la adopción (18), (en 2010 se legalizó en Argentina, en Brasil en 2013, y en Colombia en 2016) (3); aún se escuchan voces (sobre todo de sectores eclesiásticos y de partidos políticos de derecha) que expresan su oposición y temor por la motivación de las parejas homosexuales para adoptar menores así como por los efectos que un tipo de crianza a cargo de dos personas del mismo sexo pueda tener en el desarrollo de los niños. Como ya se mencionó antes, este temor surge debido a que la información sobre la homoparentalidad y sobre los efectos que esta puede tener en los niños es escasa y no está disponible más que para investigadores o académicos interesados en el tema. Los datos sobre las actitudes de la población hacia la homoparentalidad son muy pocos, además de que no se cuenta con instrumentos validados que permitan recabarlos.

Como un intento de subsanar esta carencia de información y de contar con medios adecuados para generar información válida y confiable tanto de las actitudes hacia las familias homoparentales como de las creencias de las personas sobre los efectos de la crianza por parejas del mismo sexo, se planteó el desarrollo de este estudio cuyo objetivo fue determinar la validez de constructo y la consistencia interna de las escalas de Actitudes Frente a las Familias Homoparentales (AFFH); y Creencias Acerca del Ajuste de los Niños de Familias Homoparentales (CANFH), desarrolladas ambas por investigadores españoles. Se parte del supuesto de que conocer las actitudes de las personas hacia la homoparentalidad, además de que puede aportar datos al escaso conocimiento sobre el tema, es fundamental para tener un acercamiento a cómo están viviendo las personas, los cambios que en cuestión de configuración familiar están ocurriendo, para que de ahí se pueda incidir sobre aquello que estuviera generando miedo o desinformación y que pudiera verse reflejado en el rechazo a lo que se desconoce, en este caso, una configuración parental distinta. En este sentido, los datos que se puedan generar a partir de la aplicación de escalas validadas como las que aquí se proponen, pueden constituir una fuente de información para la comunidad científica interesada en comprender el tema de la diversidad familiar.

\section{Metodología}

Los participantes en el estudio fueron estudiantes universitarios de diferentes licenciaturas, con edades entre
18 y 22 años de edad, seleccionados por conveniencia entre quienes se encontraban en los jardines de una universidad pública al momento de la encuesta. La aplicación se llevó a cabo entre marzo y mayo de 2013. La participación fue voluntaria y sin que hubiera ningún tipo de remuneración o estímulo de por medio. Cabe señalar que la investigación se consideró de riesgo mínimo para los participantes. Sin embargo, antes de la aplicación del cuestionario se informó a los participantes de los objetivos de la investigación y se les solicitó su consentimiento verbal. Cabe anotar que el proyecto de investigación fue evaluado y aprobado por el comité de ética e investigación de la Facultad de Medicina de la Universidad Nacional Autónoma de México.

\section{Instrumentos}

Actitudes Frente a las Familias Homoparentales.

La AFFH fue diseñada por un grupo de investigadores españoles de la Universidad Jaume-I en Castellón España (19). Se compone de 20 reactivos tipo Likert (que van del 1 totalmente en desacuerdo, al 5 totalmente de acuerdo) que buscan evaluar las actitudes frente a las familias homoparentales. Puntajes altos en la escala se consideran reflejo de actitudes desfavorables. Los autores señalan que la AFFH fue diseñada para medir el pensamiento, la conducta y la norma subjetiva de las actitudes hacia las familias homoparentales. El proceso de validación de la escala original consistió en acuerdo entre expertos y no se han encontrado estudios posteriores en los que se presenten otras propiedades psicométricas de la escala.

\section{Creencias Acerca del Ajuste de los Niños de Familias Homoparentales.}

Esta escala, desarrollada y validada en población española por investigadores de la Universidad de Valencia(20), tiene como objetivo medir las creencias de los sujetos sobre los efectos de la crianza por padres homosexuales en el desarrollo psicológico y social de sus hijos. La CANFH consta de 14 reactivos tipo Likert, con cinco opciones de respuesta que van del 1 totalmente en desacuerdo al 5 totalmente de acuerdo, organizados en dos subescalas: Oposición Normativa (ON). Trata de identificar las actitudes de rechazo hacia los padres homosexuales que pasan más desapercibidas por su normalización, como es el caso de la creencia de que los niños sufrirán de rechazo social. En otras palabras, la subescala operacionaliza situaciones que los ciudadanos han internalizado como "normales", "naturales" o "aceptables", no siendo conscientes de su propio prejuicio. Oposición Individual (OI): identifica indicadores personales de rechazo abierto y más agresivo hacia la crianza por padres homosexuales y por ello presenta mayor relación con las escalas tradicionales de prejuicio o rechazo hacia los homosexuales. En la escala general y en ambas subescalas, puntajes altos corresponden a actitudes desfavorables. La validación de esta escala se llevó a cabo en una muestra española de 212 estudiantes heterosexuales de psicología, encontrándose una estructura factorial de dos componentes, 
con coeficientes de consistencia interna de $\alpha=0.87$ para OI y de $\alpha=0.88$ para $\mathrm{ON}$, y con una varianza explicada de 44.48 y 14.73 respectivamente. En un estudio posterior (21), se validó la escala en una muestra de 283 estudiantes universitarios heterosexuales. Los resultados confirmaron la estructura factorial de dos subescalas e indicaron una consistencia interna de $\alpha=0.94$ para la escala total; $\alpha=0.91$ para OI y $\alpha=0.91$ para ON.

\section{Procedimiento}

Como se mencionó anteriormente, ambas escalas fueron diseñadas en España, y si bien las versiones originales no contienen términos ajenos al español que se habla en México, se siguió el procedimiento de adaptación semántica siguiendo los lineamientos de la OMS (22). En esta etapa participaron 60 estudiantes a los cuales se les solicitó que leyeran los reactivos de ambas escalas y señalaran si había algún término que no comprendieran o si la redacción era confusa. En esta etapa sólo hubo una estudiante que señaló que la palabra lesbiana le parecía ofensiva, por lo demás no hubo problemas en la comprensión del lenguaje. Una vez realizada la validación semántica, y para determinar la validez de constructo y la consistencia interna, la CANFH se aplicó a 170 participantes: 78 (46\%) hombres, 92 (54\%) mujeres (Media de edad: $18.4 \mathrm{DE}+0.94)$ en el mes de marzo, y la AFFH a 88 estudiantes: 35 (40\%) hombres, 53 (60\%) mujeres (Media de edad: 18.2 DE+0.84), en el mes de mayo (ambas en el año de 2013). A los participantes se les pidió contestar un cuestionario, se les explicó el objetivo del estudio (conocer lo que pensaban acerca de las familias de personas del mismo sexo), se les aclaró que su participación sería anónima y que sus datos serían confidenciales; y en caso de dar su consentimiento informado verbalmente, se les entregó el cuestionario, para su respectivo diligenciamiento, que una vez llenado se metió en un sobre sin revisar las respuestas enfrente de los participantes. Una vez aplicados todos los cuestionarios, se integró y analizó la base de datos con el paquete estadístico IBM SPSS Statistics 19.

\section{Resultados}

Escala de actitudes frente a las familias homoparentales (AFFH).

Se llevó a cabo un análisis factorial exploratorio de componentes principales. Los valores de adecuación muestral Kaiser - Meyer - Olkin $(\mathrm{KMO}=0.850)$ y del test de esfericidad de Bartlett $(\mathrm{X} 2=861.533, \mathrm{p}=0.0001)$ indicaron que la muestra contaba con las propiedades necesarias para llevar a cabo el análisis de validez factorial. Se utilizó el método de rotación varimax, con Eigenvalues $>1$, cargas factoriales $>0.40$ y un máximo de 250 interacciones por convergencia. Se obtuvo como resultado una estructura de 3 factores explicativos de $54.2 \%$ de la varianza; sin embargo, estos no correspondieron con la estructura propuesta por los autores o a alguna que pudiera hacer sentido teórico (los reactivos estaban mezclados), por lo cual se recurrió correr otro factorial con la instrucción de extraer 2 factores (para observar si se podría mantener la subescala de norma subjetiva). Esta organización de los reactivos en dos factores pudo dar cuenta de $46.1 \%$ de varianza explicada (Tabla 1), junto a la agrupación de los reactivos, con sus respectivas cargas factoriales (Tabla 2).

Se eliminaron tres reactivos de la escala original (16. El sistema educativo debería tener programas específicos para hijos/as de familias homoparentales, 17. Me gustaría que mi hijo/a supiera lo que es una familia homoparental, y 19. Si los niños/as pudieran elegir a la familia que los adoptará, elegirían a una familia homoparental), dado que no cargaron en ninguno de los factores. Se obtuvieron dos factores, uno denominado norma subjetiva que podría definirse como aquello que socialmente se comparte con respecto a las creencias sobre las familias homoparentales y que se cree que es verdad (Tabla 2). El otro factor, al que se denomina pensamientos y conducta, agrupa reactivos relacionados con lo que de manera personal se cree que se haría si se tuviera que tomar una decisión acerca de diferentes aspectos relacionados con las familias homoparentales.

El análisis de consistencia interna se llevó a cabo considerando sólo los 17 reactivos resultantes del análisis

Tabla 1. Actitudes Frente a las Familias Homoparentales (AFFH)

\begin{tabular}{|c|c|c|c|c|c|c|c|c|c|}
\hline \multicolumn{10}{|c|}{$\begin{array}{l}\text { Análisis factorial } \\
\qquad \mathrm{N}=88\end{array}$} \\
\hline \multirow[t]{2}{*}{ Componente } & \multicolumn{3}{|c|}{$\begin{array}{l}\text { Autovalores } \\
\text { iniciales }\end{array}$} & \multicolumn{3}{|c|}{$\begin{array}{c}\text { Sumas de las saturaciones al } \\
\text { cuadrado de la extracción }\end{array}$} & \multicolumn{3}{|c|}{$\begin{array}{l}\text { Suma de las saturaciones al } \\
\text { cuadrado de la rotación }\end{array}$} \\
\hline & Total & $\begin{array}{c}\% \text { de la } \\
\text { varianza }\end{array}$ & $\begin{array}{c}\% \\
\text { acumulado }\end{array}$ & Total & $\begin{array}{l}\% \text { de la } \\
\text { varianza }\end{array}$ & $\begin{array}{c}\% \\
\text { acumulado }\end{array}$ & Total & $\begin{array}{c}\% \text { de la } \\
\text { varianza }\end{array}$ & $\begin{array}{c}\% \\
\text { acumulado }\end{array}$ \\
\hline Norma subjetiva & 7.54 & 37.69 & 37.69 & 7.54 & 37.69 & 37.69 & 5.82 & 29.1 & 29.1 \\
\hline $\begin{array}{l}\text { Pensamiento y } \\
\text { conducta }\end{array}$ & 1.68 & 8.43 & 46.13 & 1.68 & 8.43 & 46.13 & 3.40 & 17.03 & 46.13 \\
\hline
\end{tabular}

Fuente: Elaboración propia de los autores 
Tabla 2. Actitudes Frente a las Familias Homoparentales (AFFH)

\begin{tabular}{|c|c|c|}
\hline \multicolumn{3}{|l|}{$\begin{array}{l}\text { Matriz de componentes rotados } \\
\qquad \mathrm{N}=88\end{array}$} \\
\hline \multirow[t]{2}{*}{ Reactivo } & \multicolumn{2}{|c|}{$\begin{array}{l}\text { Cargas factoriales } \\
\text { Componente }\end{array}$} \\
\hline & $\begin{array}{c}\text { Norma } \\
\text { subjetiva }\end{array}$ & $\begin{array}{l}\text { Pensamiento } \\
\text { y conducta }\end{array}$ \\
\hline 7. Si fuera juez/a permitiría el matrimonio y adopción entre homosexuales & 0.79 & \\
\hline $\begin{array}{l}\text { 2. Las parejas homosexuales (formadas por dos hombres o por dos mujeres) no } \\
\text { deberían tener hijos }\end{array}$ & 0.77 & \\
\hline $\begin{array}{l}\text { 5. Estoy a favor de que las parejas homosexuales (de hombres o de mujeres) } \\
\text { tengan derecho a la adopción }\end{array}$ & 0.77 & \\
\hline $\begin{array}{l}\text { 6. Me preocuparía que un niño/a fuera adoptado/a por una pareja homosexual (de } \\
\text { hombres o de mujeres) }\end{array}$ & 0.76 & \\
\hline $\begin{array}{l}\text { 15. Los hijos de familias homoparentales aportan a sus compañeros valores } \\
\text { positivos }\end{array}$ & 0.73 & \\
\hline 1. Los padres homosexuales pueden provocar la homosexualidad en sus hijos & 0.67 & \\
\hline 3. El hijo/a de una pareja homosexual desarrollará una autoestima baja & 0.65 & \\
\hline 13. Los padres/madres homosexuales proporcionan valores positivos a sus hijos & 0.62 & \\
\hline $\begin{array}{l}\text { 18. Los hijos de familias homoparentales tienen problemas de inserción en la } \\
\text { sexualidad }\end{array}$ & 0.51 & \\
\hline $\begin{array}{l}\text { 4. Los hijos/as de lesbianas y gays tienen un desarrollo normal de su identidad de } \\
\text { género (saber que pertenecen al género masculino o femenino) }\end{array}$ & 0.46 & \\
\hline $\begin{array}{l}\text { 20. Una familia homoparental nunca podrá satisfacer todas las áreas de desarrollo } \\
\text { de sus hijos/as al igual que una familia heteroparental }\end{array}$ & 0.45 & \\
\hline $\begin{array}{l}\text { 12. Las familias homoparentales educan peor a sus hijos/as que las familias } \\
\text { heteroparentales }\end{array}$ & & 0.81 \\
\hline $\begin{array}{l}\text { 8. La forma de vivir de las familias homosexuales es peor que la de las familias } \\
\text { heterosexuales }\end{array}$ & & 0.76 \\
\hline $\begin{array}{l}\text { 10. Si fuera maestro/a no me gustaría tener alumnos/as provenientes de familias } \\
\text { homoparentales }\end{array}$ & & 0.69 \\
\hline $\begin{array}{l}\text { 11. Si fuera padre/madre me gustaría que mi hijo/a conociera la diversidad y la } \\
\text { aceptara }\end{array}$ & & 0.60 \\
\hline $\begin{array}{l}\text { 14. Permitiría que mi hijo/a fuese a jugar a casa de un niño/a cuyos padres/madres } \\
\text { son homosexuales }\end{array}$ & & 0.60 \\
\hline 9. No me causa problemas pensar en tener un amigo/a de padres homosexuales & & 0.52 \\
\hline Reactivos inversos: 4, 5, 7, 9, 11, 13, 14 y 15 & & \\
\hline Método de extracción: Análisis de componentes principales. Método de rota & & \\
\hline
\end{tabular}

Fuente: Elaboración propia de los autores

factorial; el Alpha de Cronbach obtenido (Escala general $\alpha=$ 0.912; Pensamiento y conducta $\alpha=0.816$; Norma subjetiva $\alpha$ $=0.891$ ) reflejó niveles adecuados de homogeneidad. La correlación de los reactivos con la escala total resultó ser la adecuada por lo que no hubo necesidad de eliminar ninguno de ellos. Una vez obtenida la estructura factorial de la escala y el análisis de consistencia interna, se compararon los puntajes promedio obtenidos por hombres y mujeres (Tabla 3).

Se encontraron diferencias significativas entre los puntajes promedio de hombres y mujeres $(\mathrm{p}<0.05)$, pero sólo para la subescala de Norma subjetiva, siendo los hombres quienes obtuvieron puntajes más altos comparados con las mujeres.

Creencias acerca del Ajuste de los Niños de Familias Homoparentales(CANFH). En lo que respecta a la CANFH, los resultados de las medidas de adecuación muestral Kaiser - Meyer - Olkin $(\mathrm{KMO}=0.923)$ y la prueba de esfericidad de Bartlett $(\mathrm{X} 2=1648.5, \mathrm{p}=0.0001)$ señalaron que la muestra de estudio ( $\mathrm{N}=170)$ fue suficiente y con las características necesarias para llevar a cabo el análisis de validez factorial. Se empleó el método de extracción de 
Tabla 3. Actitudes Frente a las Familias Homoparentles (AFFH)

\begin{tabular}{|c|c|c|c|c|}
\hline \multicolumn{5}{|c|}{ Puntajes promedio. Comparación entre sexos } \\
\hline \multicolumn{5}{|c|}{$\mathrm{N}=88$} \\
\hline Subescala & Hombres & Mujeres & $t$ & $\mathrm{p}$ \\
\hline \multicolumn{5}{|l|}{ Norma subjetiva } \\
\hline Media & 41.43 & 33.07 & 2.18 & 0.03 \\
\hline DE & 7.01 & 12.49 & & \\
\hline \multicolumn{5}{|c|}{ Pensamiento / conducta } \\
\hline Media & 27.0 & 23.79 & 1.70 & 0.10 \\
\hline $\mathrm{DE}$ & 2.94 & 6.87 & & \\
\hline \multicolumn{5}{|c|}{$\begin{array}{l}\text { Rango de puntuaciones de la subescala Norma Subjetiva=11-55; Pensamiento/conducta; } \\
6-35 .\end{array}$} \\
\hline Valores altos $=$ Actitud & & & & \\
\hline
\end{tabular}

Fuente: Elaboración propia de los autores

Tabla 4. Creencias acerca del Ajuste de los Niños de Familias Homoparentales (CANFH)

\begin{tabular}{|c|c|c|c|c|c|c|c|c|c|}
\hline \multicolumn{10}{|c|}{$\begin{array}{l}\text { Análisis factorial } \\
\qquad \mathrm{N}=170\end{array}$} \\
\hline \multirow[t]{2}{*}{ Componente } & \multicolumn{3}{|c|}{$\begin{array}{c}\text { Autovalores } \\
\text { iniciales }\end{array}$} & \multicolumn{3}{|c|}{$\begin{array}{l}\text { Sumas de las saturaciones al } \\
\text { cuadrado de la extracción }\end{array}$} & \multicolumn{3}{|c|}{$\begin{array}{l}\text { Suma de las saturaciones al } \\
\text { cuadrado de la rotación }\end{array}$} \\
\hline & Total & $\begin{array}{c}\% \text { de la } \\
\text { varianza }\end{array}$ & $\begin{array}{c}\% \\
\text { acumulado }\end{array}$ & Total & $\begin{array}{l}\% \text { de la } \\
\text { varianza }\end{array}$ & $\begin{array}{c}\% \\
\text { acumulado }\end{array}$ & Total & $\begin{array}{c}\% \text { de la } \\
\text { varianza }\end{array}$ & $\begin{array}{c}\% \\
\text { acumulado }\end{array}$ \\
\hline $\begin{array}{l}\text { Oposición } \\
\text { normativa }\end{array}$ & 7.76 & 55.46 & 55.46 & 7.76 & 55.46 & 55.46 & 4.62 & 33.01 & 33.01 \\
\hline $\begin{array}{l}\text { Oposición } \\
\text { individual }\end{array}$ & 1.40 & 10.02 & 65.49 & 1.4 & 65.49 & 65.49 & 4.54 & 32.47 & 65.49 \\
\hline
\end{tabular}

Fuente: Elaboración propia de los autores

componentes principales con rotación varimax (valores Eigen $>1$ ), con cargas factoriales iguales o mayores a $0.40 \mathrm{y}$ con un máximo de 250 interacciones por convergencia. El resultado fue una estructura de dos factores que explican $65.49 \%$ de la varianza (Tabla 4), junto a la agrupación de los reactivos, con sus respectivas cargas factoriales (Tabla 5).

Como puede observarse, la aplicación a población estudiantil mexicana dio como resultado una organización de los reactivos igual a los de la escala original. Los coeficientes de consistencia interna obtenidos tanto para la escala general como para cada una de las subescalas indicaron niveles adecuados de homogeneidad (Escala general $\alpha=0.936$; Subescala de Oposición individual $\alpha=$ 0.905 y Subescala de Oposición normativa $\alpha=0.903$ ).

También para esta escala se compararon los puntajes promedio entre sexos (Tabla 6) y no se encontraron diferencias estadísticamente significativas entre ellos, aunque si es posible observar que las puntuaciones en la subescala de Oposición normativa son más altas que en Oposición individual para ambos sexos.

\section{Discusión}

Los resultados de validez y consistencia interna de las escalas AFFH y CANFH indican que ambas cuentan con las propiedades psicométricas adecuadas para medir las actitudes de estudiantes universitarios mexicanos hacia la homoparentalidad. En la validación psicométrica de ambas escalas se encontraron coeficientes de consistencia interna con niveles adecuados de homogeneidad(23), lo que indica que son escalas confiables tal como ya se ha encontrado en estudios previos con poblaciones distintas $(20,21)$, para medir las actitudes hacia las familias homoparentales en población mexicana. En lo que respecta a la validez de la CANFH, el análisis factorial confirmó la estructura propuesta por los autores(20). En el caso de la AFFH no fue posible identificar una estructura factorial similar a la de la escala original, sin embargo, la forma de 17 reactivos resultó ser bastante sólida para medir las actitudes hacia las familias homoparentales. Sobre esta escala es importante resaltar que si bien no se pudo verificar su estructura factorial, dado que como se mencionó antes los autores sólo señalan que mide tres áreas (pensamiento, conducta y 
Tabla 5. Creencias acerca del Ajuste de los Niños de Familias Homoparentales (CANFH)

\begin{tabular}{|c|c|c|}
\hline \multicolumn{3}{|l|}{$\begin{array}{l}\text { Matriz de componentes rotados } \\
\qquad \mathrm{N}=170\end{array}$} \\
\hline \multirow[t]{2}{*}{ Reactivo } & \multicolumn{2}{|c|}{$\begin{array}{l}\text { Cargas factoriales } \\
\text { Componente }\end{array}$} \\
\hline & $\begin{array}{l}\text { Oposición } \\
\text { normativa }\end{array}$ & $\begin{array}{l}\text { Oposición } \\
\text { individual }\end{array}$ \\
\hline $\begin{array}{l}\text { 10. Probablemente el niño criado por padres homosexuales no será escogido } \\
\text { como líder por sus compañeros de clase o amigos }\end{array}$ & 0.60 & \\
\hline $\begin{array}{l}\text { 12. Si los padres son homosexuales será difícil que los hijos sean invitados a } \\
\text { las fiestas de sus amigos }\end{array}$ & 0.62 & \\
\hline $\begin{array}{l}\text { 3. Es más probable que un niño sufra aislamiento social si sus amigos saben } \\
\text { que sus padres o madres son homosexuales }\end{array}$ & 0.71 & \\
\hline $\begin{array}{l}\text { 14. Lo más común es que el niño oculte la orientación sexual de sus padres a } \\
\text { sus amigos por el temor al rechazo social }\end{array}$ & 0.74 & \\
\hline $\begin{array}{l}\text { 6. Un niño adoptado por una pareja de homosexuales será objeto de broma y } \\
\text { rechazo por parte de sus compañeros }\end{array}$ & 0.79 & \\
\hline $\begin{array}{l}\text { 8. Seguramente los compañeros rechazarán a un niño cuyo padre o madre es } \\
\text { homosexual }\end{array}$ & 0.81 & \\
\hline $\begin{array}{l}\text { 9. Un niño que es educado por una pareja de homosexuales sufrirá las burlas } \\
\text { de sus compañeros }\end{array}$ & 0.86 & \\
\hline $\begin{array}{l}\text { 11. Cuando un niño manifiesta conductas homosexuales sería conveniente } \\
\text { llevarlo al psicólogo }\end{array}$ & & 0.57 \\
\hline 13. Un niño educado por madres lesbianas será un niño afeminado & & 0.67 \\
\hline $\begin{array}{l}\text { 2. En general, los niños criados por padres homosexuales tendrán más } \\
\text { problemas que los que son criados por un padre y una madre }\end{array}$ & & 0.69 \\
\hline $\begin{array}{l}\text { 7. Si un niño es adoptado por una pareja de homosexuales seguramente } \\
\text { tendrá problemas psicológicos en el futuro }\end{array}$ & & 0.74 \\
\hline $\begin{array}{l}\text { 5. Si queremos defender los intereses de los niños, sólo las parejas } \\
\text { heterosexuales deberían poder adoptar }\end{array}$ & & 0.77 \\
\hline $\begin{array}{l}\text { 4. Si los niños son criados por homosexuales tendrán más problemas de } \\
\text { confusión con su propia identidad sexual que si son criados por un padre y } \\
\text { una madre }\end{array}$ & & 0.81 \\
\hline $\begin{array}{l}\text { 1. En general, el desarrollo social de un niño es mejor cuando es educado por } \\
\text { un padre y una madre y no por una pareja de homosexuales }\end{array}$ & & 0.85 \\
\hline Método de extracción: Análisis de componentes principales. Método de rotación: No & alización V & ax con Kaiser \\
\hline
\end{tabular}

Tabla 6. Creencias acerca del Ajuste de los Niños de Familias Homoparentales (CANFH) Puntajes promedio.

\begin{tabular}{|c|c|c|c|c|}
\hline \multicolumn{5}{|c|}{$\begin{array}{l}\text { Comparación entre sexos } \\
\qquad N=170\end{array}$} \\
\hline Subescala & Hombres & Mujeres & $t$ & $p$ \\
\hline \multicolumn{5}{|c|}{ Oposición Individual } \\
\hline Media & 20.83 & 19.67 & 0.80 & 0.43 \\
\hline $\mathrm{DE}$ & 8.38 & 7.75 & & \\
\hline \multicolumn{5}{|c|}{ Oposición Normativa } \\
\hline Media & 24.32 & 23.47 & 0.93 & 0.36 \\
\hline $\mathrm{DE}$ & 6.41 & 7.41 & & \\
\hline \multicolumn{5}{|c|}{$\begin{array}{l}\text { Rango de puntuaciones de cada subescala:7 a } 35 . \\
\text { Valores altos= Actitudes desfavorables }\end{array}$} \\
\hline
\end{tabular}

Fuente: Elaboración propia de los autores 
norma subjetiva) pero no reportan datos que faciliten conocer la organización de los reactivos en ellas, la agrupación propuesta como resultado de la validación con población mexicana, además de aportar datos que posibilitan entender mejor cómo funciona esta escala, permite confirmar que para la evaluación de las actitudes un factor que no se puede soslayar y que debe ser considerado es el de la norma subjetiva, pues como lo señalan otros autores $(20,21,24)$, ésta refleja la influencia de la regulación social en las actitudes hacia las familias homoparentales.

Por otro lado, de un análisis cuidadoso del fundamento teórico de la escala CANFH propuesta por autores (20), se puede inferir que en las actitudes hay elementos aprendidos culturalmente y es a través de estos, por su carácter social, que los contenidos de las actitudes se comparten generalizan $\mathrm{y}$, normalizan. Tal normalización da como resultado que se empiece a creer que hay cosas, aspectos o elementos de la actitud que son normales, en este caso, el rechazo y las ideas erróneas acerca de la crianza de los niños por parejas del mismo sexo,--aspectos que evalúa la escala de oposición normativa-, y que no se reconocen como prejuicios o actitudes negativas; esto podría explicar por qué los puntajes de la escala de oposición normativa son más altos, además de que estaría reflejando la influencia social, o como señalan algunos autores, (20, 25-28), la norma hegemónica que privilegia a la heteroparentalidad sobre expresiones distintas como la homoparentalidad. En este sentido, y tal como lo señalan algunos autores (20, 24, 28), es interesante notar cómo los participantes atribuyen a la sociedad, esto es, a la heteronormatividad imperante, el rechazo de cualquier configuración familiar distinta de la conocida como tradicional, y lo enarbolan como elemento suficiente para negar el derecho de tener hijos a las parejas de homosexuales.

Si bien este es un estudio de validez, y dando por hecho que los datos presentados tienen como objetivo demostrar las propiedades psicométricas de las escalas; es importante retomar el papel teórico de la norma subjetiva, ya que los hallazgos sobre las diferencias de los puntajes entre sexos concuerdan con lo que diversos autores ya han señalado en el sentido de que hay una mayor proporción de hombres con actitudes desfavorables hacia la homosexualidad, comparados con sus pares mujeres (29-36). Otro autor señala que el rechazo hacia la homosexualidad es un medio que tienen los hombres de probar su identidad y rol masculinos, de manera tal que rechazar todo lo relacionado con la homosexualidad es prueba de masculinidad, pues dada la división de poderes entre hombres y mujeres, la idea popular errónea, es que los homosexuales hombres buscan adoptar un rol femenino, de ahí que sean considerados como transgresores en mayor medida puesto que adoptan un rol inferior (24). En este mismo sentido se señala que vivir en una sociedad implica asumir roles y modos de comportamiento que están conformados por las normas culturales que determinan al individuo y que estos roles obligan al hombre a demostrar su masculinidad (37-39).
Siguiendo esta línea de pensamiento, se puede hipotetizar que las diferencias entre hombres y mujeres representan la vigencia de los roles sexuales estereotipados que aún existen en nuestra sociedad y en nuestro contexto cultural donde la sexualidad aceptada y reconocida socialmente es aquella ejercida por una pareja heterosexual monogámica y unida en matrimonio. Las escalas pudieron detectar estas diferencias, lo que confirma la validez de constructo de las mismas. Algo interesante de los datos presentados es que esto sucede, pero sólo para las subescalas que tienen que ver con la norma subjetiva o influencia social en el contenido de la actitud; en otras palabras, es como si en los hombres la influencia social fuera más importante que en las mujeres, no obstante, habrá que hacer un estudio con las escalas ya validadas para observar si esto se replica en poblaciones más amplias y diversas.

Cabe señalar que una limitación del estudio fue no haber podido comparar las puntuaciones de los participantes en ambas escalas, debido a que se aplicaron a poblaciones diferentes, sin embargo, es interesante observar que se mantiene una uniformidad en cuanto a la tendencia de las puntuaciones; no obstante, se considera que en un futuro sería valioso aplicar las escalas en población no estudiantil para evaluar si se conservan las propiedades psicométricas; del mismo modo sería deseable aplicar ambas escalas en la misma población y observar las probables correlaciones entre reactivos. Otra propuesta para investigaciones venideras sería tratar de integrar ambas escalas en una sola, considerando los reactivos que se complementan, esto con la finalidad de tener cubiertos varios aspectos del fenómeno del matrimonio igualitario y la homoparentalidad.

\section{Conclusión}

Dados los datos presentados, es posible afirmar que la información recabada con ambas escalas es válida y confiable y puede ser de utilidad en el desarrollo de estudios que tengan como objetivo observar la influencia de las normas sociales en las actitudes de los estudiantes, además de que pueden ser un referente valioso puesto que en México y Latinoamérica el tema del estudio formal de la homoparentalidad apenas se está desarrollando.

\section{Conflicto de intereses}

Los autores manifiestan que no tienen ningún conflicto de interés.

\section{Referencias}

1. Swett L, Schreiber B. Danish and American students' attitudes towards gay and lesbian parenting. UW-L Journal of Undergraduate Research 2007 X: 1-5 Disponible en: https://www.uwlax.edu/urc/JURonline/PDF/2007/swett-schreiber.pdf 
2. Paternotte D. Global times, global debates? Same-sex marriage worldwide. Social Politics: International Studies in Gender, State \& Society. 2015; 22(4):653-74. DOI: https://doi.org/10.1093/sp/jxv038

3. Estos son los países que han aprobado el matrimonio homosexual en el mundo. El País, Colombia. Jueves 7 de abril de 2016;:[Acceso 20 de junio de 2016]; Disponible en: http://www.elpais.com.co/elpais/ colombia/noticias/estos-son-paises-han-aprobadomatrimonio-homosexual-mundo

4. Taub A. Fit or unfit-homosexuality and parenting. J Contemp Legal Issues. 2007; 16:29.

5. Telingator CJ, Patterson C. Children and adolescents of lesbian and gay parents. J Am Acad Child Adolesc Psychiatry. 2008;47(12):1364-8. Doi : 10.1097/CHI.0b013e31818960bc.

6. Weber S. A stigma identification framework for family nurses working with parents who are lesbian, gay, bisexual, or transgendered and their families. J Fam Nurs 2010;16(4):378-393. Doi: 10.1177/1074840710384999.

7. Fond G, Franc N, Purper-Ouakil D. Homosexual parenthood and child development: present data. Encephale 2012;38(1):10-5. Doi: 10.1016/j.encep.2011.05.005.

8. Bos HM, van Balen F, van den Boom DC. Child adjustment and parenting in planned lesbian-parent families. Am J Orthopsychiatry 2007; 77(1):38-48. DOI: 10.1037/0002-9432.77.1.38

9. Rosenfeld MJ. Nontraditional families and childhood progress through school. Demography 2010;47(3):75575.

10. Patterson CJ. Children of lesbian and gay parents. Child Dev 1992;63(5):1025-42.

11. Anderssen N, Amlie C, Ytterøy EA. Outcomes for children with lesbian or gay parents. A review of studies from 1978 to 2000. Scand J Psychol 2002;43(4):335-51.

12. Averett $P$, Nalavany B, Ryan S. An evaluation of gay/lesbian and heterosexual adoption. Adopt Q. 2009; 12(3):129-51. DOI: 10.1080/10926750903313278

13. Crouch SR, Waters E, McNair R, Power J, Davis E. ACHESS--The Australian study of child health in samesex families: background research, design and methodology. BMC public health. 2012; 13 (12): 646. Doi: 10.1186/1471-2458-12-646.

14. Kleber DJ, Howell RJ, Tibbits-Kleber AL. The impact of parental homosexuality in child custody cases: a review of the literature. The Bulletin of the American Academy of Psychiatry and the Law. 1986;14(1):81-7.

15. Stacey J, Biblarz TJ. (How) does the sexual orientation of parents matter? Am Sociol Rev. 2001;66(2):159-83.

16. Nickman SL, Rosenfeld AA, Fine P, Macintyre JC, Pilowsky DJ, Howe RA, et al. Children in adoptive families: overview and update. J Am Acad Child Adolesc P s y c h i a try $2005 ; 44(10): 987-95$. DOI:10.1097/01.chi.0000174463.60987.69

17. Paige RU. Proceedings of the American Psychological Association for the Legislative Year 2004: Minutes of the Annual Meeting of the Council of Representatives, February 20-22, 2004, Washington, DC, and July 28 and 30, 2004, Honolulu, Hawaii, and Minutes of the February, April, June, August, October, and December 2004 Meetings of the Board of Directors.American Psychologist $2005 ; 60(5): 436-511$. http://dx.doi.org/10.1037/0003-066X.60.5.436
18. Decreto por el que se reforman diversas disposiciones del código civil para el D.F. y del código de procedimientos para el D.F., Gaceta Oficial del Distrito Federal, 115, 2009. Ramírez S, Moliner V, Vicent L. Actitudes frente a las familias homoparentales en el contexto escolar. España:2006 1139-5486.

19. Ramírez S, Moliner V, Vicent L. Actitudes frente a las familias homoparentales en el contexto escolar. Universitat Jaume, Jornades de Foment de la Investigació 2006:1-23 Disponible en: http://repositori.uji.es/xmlui/bitstream/handle/10234/786 87/forum_2006_38.pdf?sequence=1

20. Frias-Navarro $\bar{D}$, Monterde-i-Bort $H$. A scale on beliefs about children's adjustment in same-sex families: Reliability and validity. J Homosex. 2012;59(9):127388.Doi: 10.1080/00918369.2012.720505.

21. Barrientos J, Cárdenas M, Gómez F, Frías-Navarro D. Assessing the dimensionality of deliefs about children's adjustment in same-sex families fcale (BCASSFS) in Chile. Sexuality Research and Social Policy. 2013;10(1):43-51. Doi: 10.1007/s13178-012-0107-0

22. World Health Organization. Process of translation and adaptation of instruments. World Health Organization [Acceso Junio 2016 ] Disponible en:http://www.who.int/ substance_abuse/research_tools/translation/en/. 2009.

23. Cervantes V. Interpretaciones del coeficiente de alpha de Cronbach. Avances en medición. 2005; 3(1): 9-28. Disponible en: http://www.humanas.unal.edu.co/ psicometria/files/1113/8574/8604/Articulo_1_Alfa_de_C ronbach 9-28 2.pdf

24. Ortiz-Hernández L. La opresión de minorías sexuales desde la inequidad de género. Polít cult. 2004; 22: 16182. Disponible en: http://www.scielo.org.mx/pdf/ polcul/n22/n22a09.pdf

25. Flores PF. Psicología social y género; el sexo como objeto de representación social. México: UNAM; 2001.

26. Serrano O, Eréndira S. Psicología social y género; el sexo como objeto de representación social. Península 2014; 9(2): 165-168. Disponible en: <http://www. scielo.org.mx/ scielo.php?script=sci_arttext\&pid=S1870-57662014000 200008\&lng=es\&nrm=iso>. ISSN 1870-5766.

27. Rodríguez CO. Las representaciones sociales: entretejidos de la razón y la cultura. Relaciones Estudios de historia y sociedad. 2003;24(93):82-95.

28. Gutmann MC. La" falocedad" de continuos: salud reproductiva entre adolescentes en Oaxaca de Juárez. Estudios Sociales 2005;13(26):117-43.

29. Morse CN, McLaren S, McLachlan AJ. The attitudes of australian heterosexuals toward same-sex parents. Journal of GLBT Family Studies. 2007;3(4):425-55.

30. Brown MJ, Henriquez E. Socio-demographic predictors of attitudes towards gays and lesbians. Individ Differ Res. 2008;6(3):193-202.

31. Herek GM. Heterosexuals attitudes toward bisexual men and women in the United States. J Sex Res 2002; 39(4): 264-74. DOI:10.1080/00224490209552150

32. Horn SS. Heterosexual adolescents' and young adults' beliefs and attitudes about homosexuality and gay and lesbian peers. Cogn Dev 2006;21(4):420-40. Disponible en: http://www.sciencedirect.com/science/article/ pii/S0885201406000657

33. Johnson $\mathrm{MH}$, Henderson P. Acquiring and demonstrating attitudes in medical education: attitudes to homosexuality as a case study. Med Teach. 2000;22(6):585-91. Doi: 
10.1080/01421590050175578.

34. Katsurada E, Sugihara Y. Gender-role identity, attitudes toward marriage, and gender-segregated school backgrounds. Sex Roles. 2002; 47(5):249-58.DOI: 10.1023/A:1021334710431

35. Korfhage BA. Psychology graduate students' attitudes toward lesbians and gay men. J Homosex 2006;51(4):145-59. DOI: 10.1300/J082v51n04_08

36. Mohipp C, Morry MM. The relationship of symbolic beliefs and prior contact to heterosexuals' attitudes toward gay men and lesbian women. Can J Behav Sci 2004; 36(1):36-44.

37. Toro-Alfonso J, Varas-Díaz N. Los otros: prejuicio y distancia social hacia hombres gay y lesbianas en una muestra de estudiantes de nivel universitario. Int J Clin Health Psychol 2004;4(3):537-51.Disponible en: http://www.aepc.es/ijchp/articulos pdf/ijchp-123.pdf

38. Campo-Arias A, Herazo E. Homofobia en estudiantes de medicina: una revisión de los diez últimos años. MedUNAB 2008;11(2):120-123.

39. Verduzco-Lozano I, Diaz-Loving R. Medición de la homofobia en México: Desarrollo y validación. RIDEP. 2010;2(30):105-24. Disponible en: http:// alfama.sim.ucm.es/wwwisis2/wwwisis.exe/[in=psylink.in $1 / ? \mathrm{mfn}=032231 \& \mathrm{campo}=\mathrm{v} 300 \& \mathrm{occ}=1$ 